\title{
Effets De La Digestion Gastrique Sur Les Propriétés Anthelminthiques De Zanthoxylum Zanthoxyloides (Lam.) Zepernick \& Timlerto Et De Newbouldia Laevis (P.Beauv.) Sur Haemonchus Contortus
}

Irvine Yèinou Minaflinou Sacca Sidi, (MA)

Géorcelin Goué Alowanou, (PhD, Assistant)

Esaïe Tchétan, (MA)

Maliki Youssouf Aminou, (MA)

Sylvie Mawulé Hounzangbé-Adoté, (PhD, Professeur Titulaire)

Université d'Abomey-Calavi / Faculté des Sciences Agronomiques, Laboratoire d'Ethnopharmacologie et de Santé Animale, Bénin

Séverin Babatoundé, (PhD, Maître de conférences)

Université d'Abomey Calavi / Faculté des Sciences Agronomiques,

Laboratoire de Zootechnie, Bénin

doi: 10.19044/esj.2017.v13n24p204 URL:http://dx.doi.org/10.19044/esj.2017.v13n24p204

\section{Abstract}

Several recent studies have shown that medicinal plants Zanthoxylum zanthoxyloides (Fagara) and Newbouldia laevis possess anthelmintic activities in vitro on different stages of development of gastrointestinal nematodes. The objective of this study was to evaluate the in vitro anthelmintic properties of residues from digestion in the rumen of leaf powders of both plants on the migration of the 3rd-stage larvae L3s of Haemonchus contortus. Residues obtained after incubation at $0 \mathrm{~h}, 24 \mathrm{~h}$ and $96 \mathrm{~h}$ kinetic points of the leaf powders of both plants in the rumen of sheep with fistulae were used for the assay. The larval migration inhibition test evaluated the anthelmintic properties of the methanolic extracts of residues of the two plants. The effect of plant extracts on larval migration was notdose-dependent $(\mathrm{p}>0.05)$ but was a function of plant incubation time ( $\mathrm{p}$ $<0.01$ ) with $N$. laevis extracts. The reduction in larval migration was dosedependent $(\mathrm{p}<0.05)$ with the extract of the not-incubated Fagara powder, with the extracts of the residues $(24 \mathrm{~h}$ and $96 \mathrm{~h})$ she was not-dose-dependent ( $\mathrm{p}>0.05)$. N. Laevis seems to have retained his anthelmintic property after incubation in sacco in the rumen. On the other hand, Fagara seems to lose its effectiveness as it stays in the rumen. Findings obtained on these plants 
confirm their traditional use in veterinary medicine especially in the control of helminthiasis.

Keywords: Newbouldia laevis, Haemonchus contortus, Zanthoxylum zanthoxyloides

\section{Résumé}

Plusieurs études récentes ont montré que les plantes médicinales Zanthoxylum zanthoxyloides (Fagara) et Newbouldia laevis possèdent des activités anthelminthiques in vitro sur différents stades de développement des nématodes gastro intestinaux. La présente étude a pour objectif d'évaluer les propriétés anthelminthiques in vitro, des résidus issus de la digestion dans le rumen des poudres de feuilles des deux plantes sur la migration des larves infestantes L3s de Haemonchus contortus. Les résidus obtenus après incubation à des points cinétiques de $0 \mathrm{~h}, 24 \mathrm{~h}$ et $96 \mathrm{~h}$ des poudres de feuilles des deux plantes dans le rumen des ovins munis de fistules ont servi au test. Le test d'inhibition de la migration larvaire a permis d'évaluer les propriétés anthelminthiques des extraits méthanoliques de résidus des deux plantes. L'effet des extraits sur la migration des larves a été non dose-dépendante ( $p$ $>0,05)$ mais fonction du temps d'incubation de la plante $(\mathrm{p}<0,01)$ avec les extraits de $N$. laevis. La réduction de la migration des larves a été dose dépendante $(p<0,05)$ avec l'extrait de la poudre de Fagara non incubée par contre, avec les extraits des résidus ( $24 \mathrm{~h}$ et $96 \mathrm{~h}$ ) elle a été non dose dépendante $(\mathrm{p}>0,05)$. N. Laevis semble avoir conservé sa propriété anthelminthique après incubation in sacco dans le rumen. Par contre Fagara semble perdre son efficacité au fur et à mesure qu'il séjourne dans le rumen. Les résultats obtenus sur les plantes confirment bien leur usage traditionnel en médicine vétérinaire notamment dans le contrôle des helminthiases.

Mots clés: Newbouldia laevis, Haemonchus contortus, Zanthoxylum zanthoxyloides

\section{Introduction}

Les parasitoses gastro-intestinales causées par les nématodes gastrointestinaux constituent des pathologies majeures chez les petits ruminants entretenus au pâturage et, peuvent parfois entraîner des pertes importantes de production (Andrea et al., 2011). Ces pertes se traduisent par la réduction du gain de poids vif, une diminution de l'ingestion alimentaire, de la production de laine et de la production laitière chez les moutons. Par ailleurs, il a été signalé que la présence de ces parasites dans le tube digestif peut entrainer l'anorexie, une augmentation du taux de protéines endogènes (albumine par exemple) dans le plasma, des pertes de sang et une production accrue de mucus (Hassanpour et al., 2011). 
Le contrôle de ces parasites a toujours été basé sur l'utilisation des molécules de synthèse qui ont fini par montrer leurs limites telles que la résistance développée par les parasites face aux anthelminthiques de synthèses, la cherté et l'inaccessibilité de ces derniers. Dans ces conditions, il devient opportun d'envisager de nouvelles méthodes de lutte antiparasitaire (Satyavir Singh et Gupta, 2010) notamment l'usage des plantes médicinales potentiellement anthelminthiques (Wabo-Poné et al., 2011). Dans les pays à revenus limités, le contrôle des helminthiases par les petits éleveurs est largement basé sur l'utilisation des plantes médicinales (Hounzangbé-Adoté et al., 2005a) dont Zanthoxylum zanthoxyloides (Fagara) et Newbouldia laevis potentiellement dotées de propriétés antiparasitaires. Pour la validation scientifique des deux plantes pour leur propriété anthelminthique, plusieurs investigations cliniques ont été entreprises débouchant sur des résultats satisfaisants. Elles se sont révélées sous différentes formes (Feuilles fraîches, poudre de feuilles extraits organiques) très efficaces sur les parasites gastrointestinaux des petits ruminants (Hounzangbé-Adoté et al., 2005a,b ; Azando et al., 2011a, Minanflinou et al., 2015). Le plus souvent ces plantes anthelminthiques sont des plantes fourragères consommées par les ruminants et par conséquent auront une double fonction de nutrition et de déparasitant regroupée sous le nom d'alicament.

Dans le but de comprendre alors l'utilisation que ces animaux font de ces plantes lorsqu'ils les consomment, des études ont été entreprises pour évaluer les propriétés anthelminthiques in vitro des résidus issus de la digestion dans le rumen des ruminants plus précisément des ovins Djallonké sur Haemonchus contortus. Ainsi, les travaux de Dédéhou (2015) sur Pterocarpus erinaceus et Parkia biglobosa ont montré que les deux plantes ne perdaient pas leurs propriétés anthelminthiques après $72 \mathrm{~h}$ d'incubation dans le rumen des animaux malgré une disparité non négligeable de la matière sèche. Ces résultats ont été confortés par les travaux de Alowanou et al., (2015) sur trois autres espèces fourragères (Mitragyna inermis, Combretum glutinosum et Bridelia ferruginea) cette fois ci en quantifiant les métabolites secondaires (Tanins condensés, flavonoïdes et phénols totaux) contenus dans les résidus des plantes obtenus après $72 \mathrm{~h}$ d'incubation dans le rumen des animaux. Les résultats ont montré que les pertes en matières sèches et logiquement en métabolites secondaires responsables des propriétés anthelminthiques n'avaient aucune influence sur l'efficacité des plantes. La question qui se dégage alors est de savoir si un temps incubation plus prolongé n'affecterait pas l'efficacité des plantes ?

C'est pour répondre à cette interrogation que la présente étude se donne comme objectif d'évaluer les propriétés anthelminthiques in vitro des résidus de Fagara et $N$. laevis issus de la digestion dans le rumen des ovins 
Djallonké sur Haemonchus contortus, un parasite hématophage à intérêts agronomiques.

\section{Matériels et méthodes \\ Récolte et préparation des plantes}

Les feuilles de Fagara et $N$. laevis ont été récoltées matures dans la commune d'Abomey-Calavi (Sud du Bénin) courant le mois de Novembre 2016. Ensuite, elles ont été identifiées et authentifiées à l'Herbier National de l'Université d'Abomey-Calavi respectivement sous les numéros : AA 6301 /HNB et AA 6302 / HNB. Les feuilles des plantes ont été séchées en salle à la température ambiante du laboratoire. Après deux semaines de séchage, elles ont été broyées au tamis de $2 \mathrm{~mm}$ de maille dans un moulin à marteau. La poudre est conservée dans un bocal hermétique à la température ambiante.

\section{Réalisation de la dégradabilité in sacco \\ * Matériel animal et rationnement}

Cinq (05) béliers adultes de race Djallonké pesant en moyenne 30 $\mathrm{kg}$ ont été utilisés. Ces animaux fistulés ont porté des canules du rumen de 4 $\mathrm{cm}$ de diamètre, lavées tous les jours avec du Dettol à $5 \%$. Durant l'expérimentation, les béliers ont été logés en stabulation entravée et ont reçu une ration d'entretien à base de Panicum maximum var. $\mathrm{C} 1$ frais et un complément (graines de coton et épluchures de manioc) à raison de $50 \mathrm{~g}$ de matière sèche/ $\mathrm{kg}$ PV distribuée en 2 repas à $8 \mathrm{~h}$ et à $16 \mathrm{~h}$. Cette ration a été distribuée aussi bien en période pré - expérimentale qu'expérimentale. La composition centésimale de la ration est la suivante : 70\% de Panicum maximum var. $\mathrm{C} 1$ frais $+30 \%$ de concentré, c'est à dire $35 \mathrm{~g} / \mathrm{kg} \mathrm{PV}$ de Panicum maximum var. $\mathrm{C} 1$ frais, $7,5 \mathrm{~g}$ matière sèche $/ \mathrm{kg} \mathrm{PV}$ de graines de coton et 7,5 g matière sèche $/ \mathrm{kg}$ PV d'épluchures de manioc par repas. Cette proportion est généralement utilisée dans les études de dégradabilité in sacco (Babatoundé, 2005). Les graines de coton ont été distribuées le matin et les épluchures de manioc l'après-midi. Les animaux ont disposé de blocs à lécher à base de sels minéraux et ont reçu de l'eau ad libitum.

\section{* Préparations des sachets et des échantillons à incuber}

Les sachets en toile de nylon de porosité $42 \mu \mathrm{m}$ et de dimensions internes : $10 \mathrm{~cm}$ x $15 \mathrm{~cm}$ ont été utilisés. Les supports des sachets ont été des tuyaux en plastiques souples de $1 \mathrm{~cm}$ de diamètre et $10 \mathrm{~cm}$ de longueur. Dans leurs bords supérieurs, les tuyaux ont été reliés par un fil en nylon de $0,60 \mathrm{~mm}$ de diamètre et de $25 \mathrm{~cm}$ de longueur pour faciliter leur retrait du rumen. Chaque tuyau a correspondu à une des espèces de plantes en études si bien qu'un animal en expérimentation a reçu deux tuyaux. Les sachets vides ont été séchés à l'étuve à $60^{\circ} \mathrm{C}$ pendant $24 \mathrm{~h}$ avant d'être pesés à l'aide d'une 
balance analytique de précision au 1/10 mg. Deux pesées ont été effectuées sur les sachets vides. Après les pesées, les sachets ont été remis à l'étuve à $60{ }^{\circ} \mathrm{C}$ pendant 30 min avant d'être pesés à nouveau. La prise d'essai a été de $5 \mathrm{~g}$ de matière broyée au tamis de $2 \mathrm{~mm}$. Les sachets ont été ensuite fermés par un collier de serrage en plastique avant leur fixation sur le tuyau.

\section{* Incubation des sachets dans le rumen}

Les deux (02) tuyaux portant les sachets ont été introduits dans le rumen juste avant les repas. Chaque tuyau a porté 3 sachets disposés en quinconce, chaque sachet représentant un temps d'incubation pour un fourrage donné. L'étude de dégradabilité in sacco a été établie sur trois points de cinétique $(0 \mathrm{~h}, 24$ et $96 \mathrm{~h})$, ceci afin de permettre une digestion complète des poudres des plantes. L'introduction des sachets a été réalisée de manière séquentielle afin de retirer en même temps les trois sachets à la fin de la période d'incubation considérée.

\section{* Récupération, lavage et pesée des sachets après incubation}

Au terme de l'incubation, les sachets ont été retirés du rumen pour l'ensemble des animaux. Le retrait s'est fait grâce au fil en nylon relié au bout du tuyau. Une fois retirés, ils ont été rincés sous jet d'eau au robinet. Après les lavages successifs, les sachets ont été séchés avec leur contenu à l'étuve à $40{ }^{\circ} \mathrm{C}$ pendant $48 \mathrm{~h}$. A la sortie de l'étuve, deux pesées ont été réalisées sur les sachets avec leur contenu et de la même manière que pour les pesées de sachets vides avant l'incubation. Après cette double pesée, le contenu des sachets a été récupéré dans un récipient en plastique pour les analyses ultérieures.

\section{Techniques de préparation des extraits}

Les résidus obtenus après incubation des poudres dans le rumen sont extraits dans une solution de méthanol dans une proportion de 70 :30 (méthanol-eau distillée) pendant une heure sous agitation magnétique à $50{ }^{\circ} \mathrm{C}$. Après filtration du mélange, le filtrat est recueilli et évaporé sous vide à l'aide d'un évaporateur rotatif. La phase aqueuse obtenue a été mise à l'étuve à $40^{\circ} \mathrm{C}$ pour l'évaporation à sec. L'extrait ainsi obtenu est pesé puis conservé au réfrigérateur à $+4{ }^{\circ} \mathrm{C}$.

\section{Activité biologique des deux plantes \\ * Extraction des larves}

Les larves infestantes ont été obtenues par coproculture à partir de matières fécales de brebis préalablement infestées artificiellement par une souche pure de $H$. contortus, laissées en culture à température ambiante pendant 10 jours. Les larves ont ensuite été extraites de la masse fécale par le dispositif de Baermann dont le principe repose sur l'hygrotropisme.

\section{Test de migration larvaire}


Le test appliqué repose sur la mesure du taux de migration des larves du parasite à travers une membrane après contact avec les extraits à tester. Le pourcentage de larves ayant traversé la membrane permet de calculer l'inhibition de la migration larvaire (LMI) associée aux extraits de plantes (Rabel et al., 1994). Une quantité connue de larves $\mathrm{L}_{3 \mathrm{~s}}\left(1000 \mathrm{~L}_{3 \mathrm{~s}} / \mathrm{mL}\right)$ est mise en contact pendant $3 \mathrm{~h}$ à $23{ }^{\circ} \mathrm{C}$ avec chaque extrait à tester à différentes concentrations $(1200,600,300$ et $150 \mu \mathrm{g} / \mathrm{mL})$ à raison de 3 répétitions par concentration. Un témoin négatif (tampon $\mathrm{PBS}, \mathrm{pH} 7$ et 0,15 M) a permis d'évaluer la migration des larves en absence de la plante. Un témoin positif (lévamisole 125, 250 et $500 \mu \mathrm{g} / \mathrm{mL}$ ) a été également constitué. Les larves $\mathrm{L}_{3 \text { s }}$ sont ensuite rincées 3 fois et centrifugées, puis laissées en migration à travers des mailles de $20 \mu \mathrm{m}$ de diamètre pendant $3 \mathrm{~h}$ à une température de $23{ }^{\circ} \mathrm{C}$. Les larves ayant migré sont reprises dans un volume de 1,5 mL. Le nombre de larves est alors compté dans $200 \mu \mathrm{l}$. Le pourcentage d'inhibition de la migration larvaire a été calculé à l'aide de la formule suivante :

$$
L M I=\frac{T-M}{T} x 100
$$

Où $\mathrm{T}$ le nombre total de $\mathrm{L}_{3 \text { s }}$ ayant été en contact du PBS et $\mathrm{M}$ le nombre de $\mathrm{L}_{3}$ en contact avec les extraits.

\section{Analyses statistiques}

Les différentes valeurs de la migration des larves infestantes ont été intégrées dans un modèle d'analyse de variance sur mesure répété à deux facteurs (temps d'incubation, résidu de la plante testée) exécuté dans le logiciel R (R Core Team, 2013). La comparaison des différentes moyennes a été faite à l'aide de la procédure SNK qui exécute le test Student-NewmanKeuls(SNK) à l'aide du package agricolae (Mendiburu, 2013) du logiciel R (RCore Team 2013). Les moyennes générées ont servi à construire les graphes d'illustration. Les différences sont considérées significatives au seuil de $5 \%$.

\section{Résultats}

\section{Pourcentage de disparition de la matière sèche}

Les pourcentages de disparition de la matière sèche de Fagara et de N.laevis en fonction du temps d'incubation dans le jus de rumen des ovins Djallonké sont présentés par la figure 1. 


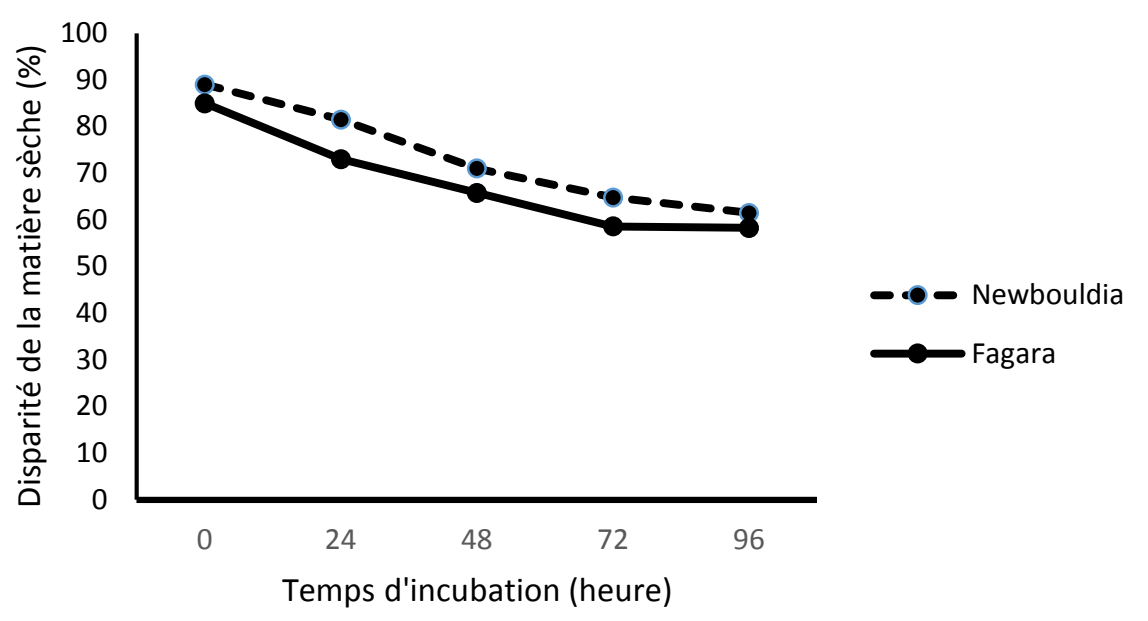

Figure 1 : Courbe de disparité de la matière sèche de Fagara et de N. laevis dans le rumen.

La disparition de la matière sèche a varié en fonction du temps d'incubation de la poudre de feuilles des plantes $(\mathrm{p}<0,05)$ mais pas suivant la poudre de feuilles des plantes incubées $(\mathrm{p}>0,05)$. Cette disparition a été entre 0 h et 96 h de 30,7\% pour $N$. laevis et de 26,7\% pour Fagara.

Effets de l'extrait méthanolique des résidus des deux plantes issues de la digestion sur la migration des larves $(\mathrm{L} 3 \mathrm{~s})$ de $\boldsymbol{H}$. contortus

L'action inhibitrice des plantes sur la migration des larves infestantes de $H$. contortus a été fonction du temps d'incubation des plantes $(\mathrm{p}<0,001)$ d'une part et non dose dépendante $(\mathrm{p}>0,05)$ d'autre part (Figures 2,3 et 4).

L'extrait de la poudre simple de $N$. laevis excepté la dose faible dose de $75 \mu \mathrm{g} / \mathrm{mL}$ a inhibé à plus de $40 \%$ la migration des larves de $H$. contortus in vitro quel que soit le temps d'incubation dans le rumen des animaux (Figure 2). Les extraits des résidus de $N$. laevis obtenus après $24 \mathrm{~h}$ et $96 \mathrm{~h}$ ont connu le même taux de réduction de la migration des larves seulement aux fortes doses de 1200 et $600 \mu \mathrm{g} / \mathrm{mL}$ (Figure 2). A faible dose de $75 \mu \mathrm{g} / \mathrm{mL}$ les résidus obtenus après $96 \mathrm{~h}$ d'incubation dans le rumen des animaux sont moins efficaces $(p>0,05)$ que ceux obtenus après $24 \mathrm{~h}$. 


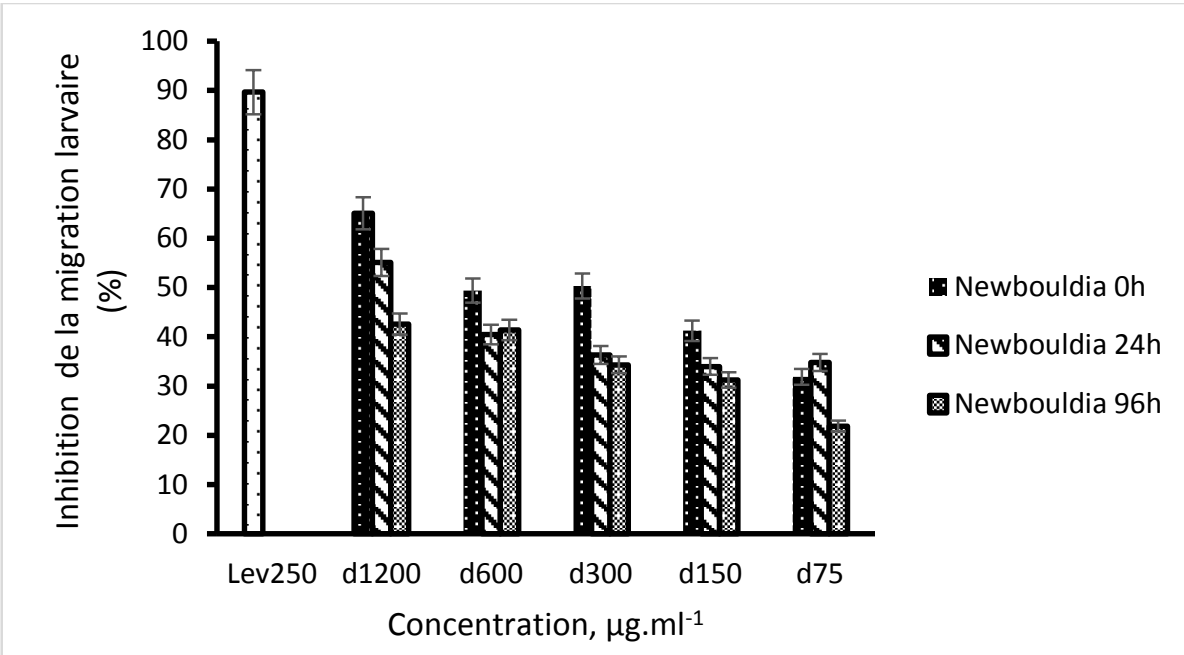

Figure 2 : Effets de l'extrait méthanolique de la poudre simple et des résidus d'incubation de $N$. laevis sur la migration larvaire de $H$. contortus.

Aux doses fortes de 1200 et $600 \mu \mathrm{g} / \mathrm{ml}$ et la dose moyenne de 300 $\mu \mathrm{g} / \mathrm{mL}$, l'extrait de la poudre simple (Fagara $0 \mathrm{~h}$ ) et les résidus de Fagara obtenus après $24 \mathrm{~h}$ d'incubation ont fortement inhibé la migration des larves $(\mathrm{p}<0,01)$ avec une forte sensibilité des larves à l'extrait de la poudre simple (Figure 3). Aux faibles doses (150 et $75 \mu \mathrm{g} / \mathrm{mL}$ ), la réduction de la migration par les extraits de la poudre simple (Fagara $0 \mathrm{~h}$ ) et des résidus de $24 \mathrm{~h}$ a été aussi non négligeable $(\mathrm{p}<0,05)$ et comparable à l'effet de l'extrait des résidus de Fagara obtenus après $96 \mathrm{~h}$ d'incubation aux fortes doses (Figure $3)$. Aux faibles doses néanmoins, les résidus de $24 \mathrm{~h}$ ont plus réduit $(\mathrm{p}<0,05)$ la migration des larves que les résidus de $96 \mathrm{~h}$.

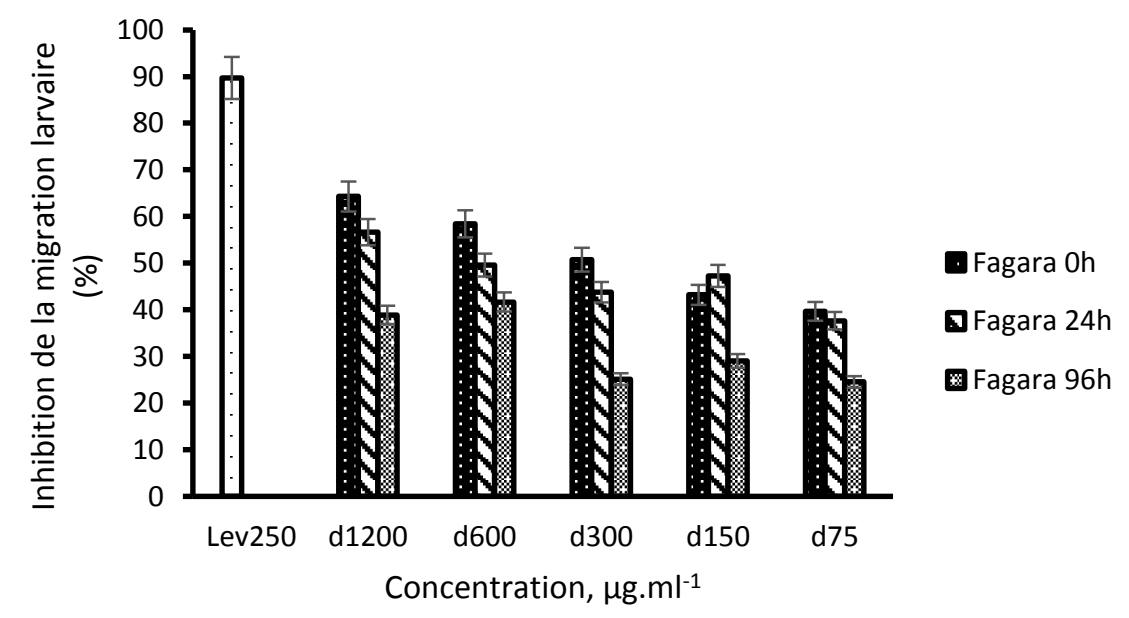

Figure 3 : Effets de l'extrait méthanolique de la poudre simple et des résidus d'incubation de Fagara sur la migration larvaire de $H$. contortus. 
La comparaison des différents effets inhibiteurs des résidus de $24 \mathrm{~h}$ et de $96 \mathrm{~h}$ des deux plantes montre que quel que soit la dose et la plante, les résidus de $24 \mathrm{~h}$ ont plus réduit $(\mathrm{p}<0,05)$ la mobilité des larves de $H$. contortus que les résidus de 96 h (Figure 4). Egalement les résidus de Fagara ont semblé être plus efficaces que ceux de $N$. laevis même si la différence n'a pas été significative.

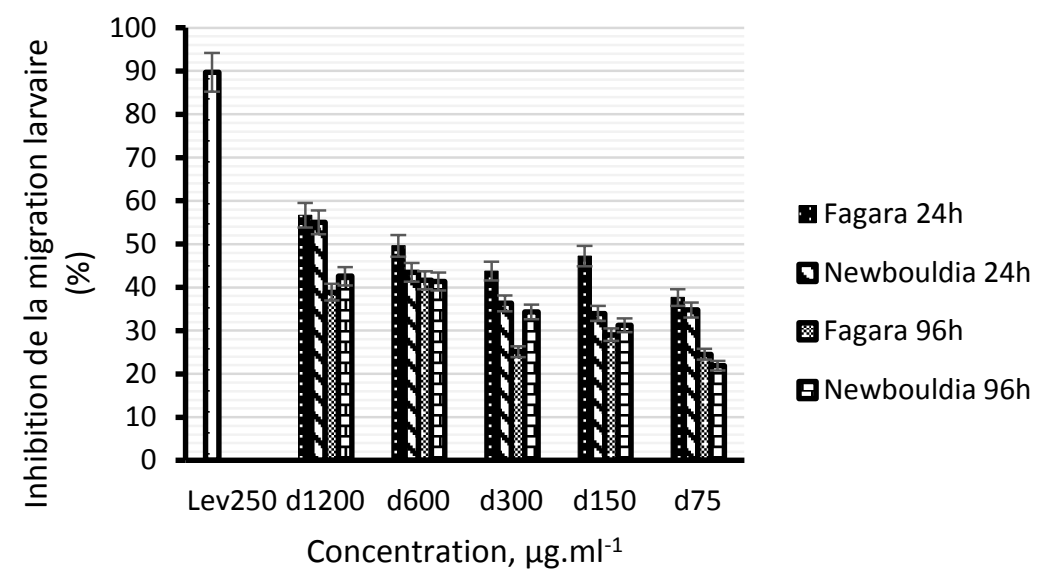

Figure 4 : Effets comparés de l'extrait méthanolique des résidus d'incubation de Fagara et de $N$. laevis sur la migration larvaire de $H$. contortus.

\section{Discussion}

Fagara et $N$. laevis sont des plantes tropicales largement étudiées pour leurs propriétés anthelminthiques sur les parasites gastro-intestinaux des petits ruminants. Les deux plantes se sont déjà révélées efficaces sur les trois stades de développement de $H$. contortus et de Trichostrongylus colubriformis in vitro (Hounzangbé-Adoté et al., 2005a, b) et in vivo chez les ovins et les caprins (Hounzangbé-Adoté et al., 2005c ; Azando et al., 2011a ; Minanflinou et al., 2015). Toutes ces études réalisées prouvent que les deux plantes peuvent être utilisées comme alternative ou en complément aux anthelminthiques de synthèse. Alors il est question de savoir si la digestion ruminale des deux plantes fourragères dans les conditions naturelles ne réduirait pas l'efficacité de ces plantes ?

Le test de migration larvaire utilisé dans cette étude pour évaluer l'effet antiparasitaire des résidus des plantes est un test rapide et moins couteux pour la détermination de l'effet des substances qui pourraient causer la paralysie des nématodes gastro-intestinaux (D'Assonville et al., 1996). Les extraits du résidu des plantes issues de la digestion dans le rumen ont significativement à forte dose et au temps d'incubation de $24 \mathrm{~h}$ inhibé la migration des larves de $H$. contortus. Cette inhibition a été non dose dépendante avec une efficacité beaucoup plus prouvée de Fagara. Les plantes conserveraient donc leurs propriétés anthelminthiques après $24 \mathrm{~h}$ 
d'incubation. Cette efficacité diminuerait ainsi en fonction des temps d'incubation dans le rumen puisque les résidus de $96 \mathrm{~h}$ ont été les moins efficaces. Ce fait est justifié par le fort taux de disparité de la matière sèche enregistré chez les deux plantes à la fin de l'incubation des plantes dans le jus de rumen des animaux au temps $96 \mathrm{~h}$. La forte activité des microorganismes du rumen aurait favorisé une forte perte des métabolites secondaires comme les tanins condensés, les flavonoïdes, les alcaloïdes (Olounladé, 2005) et les terpènes identifiés dans les plantes et reconnus être responsables des propriétés anthelminthiques de ces dernières (Azando et al., 2011b). En effet, chez les ruminants, le rumen est un compartiment doté d'un écosystème qui abrite plusieurs types de microorganismes majoritairement anaérobies ou anaérobies facultatives, et qui se caractérise par un $\mathrm{pH}$ proche de la neutralité (6 à 7) (Brunet, 2008). Ainsi, lorsque les tanins condensés sont ingérés, ils se fixent aux protéines salivaires (en particulier avec les protéines riches en proline) ou alimentaires (Butter et al., 1999) aux enzymes bactériennes (Brunet, 2008) et forment avec elles des complexes stables au pH du rumen (Aerts et al., 1999).

Mieux dans une étude récente, Alowanou et al. (2015) ont montré qu'un temps de digestion étendu des plantes, $M$. inermis, $C$. glutinosum et $B$. ferruginea dans le rumen des animaux induisait une perte des tanins condensés, des flavonoïdes et des phénols totaux. Mais ces auteurs n'ont pas enregistré une baisse significative de l'efficacité des trois plantes après un temps d'incubation étendu de $72 \mathrm{~h}$. On pourrait supposer qu'au-delà de $72 \mathrm{~h}$ d'incubation comme le cas de la présente étude, l'efficacité des trois plantes baisserait significativement. Aussi on doit prendre en compte le degré de dégradabilité de la matière sèche propre à chaque plante fourragère.

La diminution de la migration des larves $L_{3 s}$ par les extraits des résidus des plantes observées dans cette étude pourrait être à l'origine de la perturbation de leur installation dans la paroi de la muqueuse du tube digestif. Cette action inhibitrice de la migration des larves de H. contortus serait rendue possible grâce aux grandes familles de composés chimiques mises en évidence dans cette étude à savoir les tanins, les flavonoïdes et les phénols totaux. En effet Molan et al. (2003) et Brunet (2008) ont montré que les flavonoïdes induisaient désaltérations structurelles au niveau des larves infestantes empêchant ainsi leur migration. Les flavonoïdes et les tanins contenus dans la fraction polaire de Leuceana leucocephala (Adémola et al., 2005) ont montré un effet sur la migration des larves $\mathrm{L}_{3 s}$ de $H$. contortus. En effet selon Bruneton (1999), la stabilité de ces complexes tanins condensésprotéines devient défavorisée au $\mathrm{pH}$ acide de l'abomasum. Ainsi, les conditions de l'abomasum induisent une libération des tanins condensés fixés. Ces derniers sont par la suite disponibles dans les portions du tube digestif qui font suite au rumen, ce qui justifierait l'aptitude des plantes qui 
en contiennent à perturber la viabilité des vers adultes ou la fertilité des vers femelles (Marie-Magdeleine et al., 2010 ; Azando et al., 2011b).

\section{Conclusion}

La présente étude a mis en évidence in vitro l'action inhibitrice des extraits des résidus de Fagara et $N$. laevis après un temps d'incubation plus long dans le rumen des animaux sur la migration des larves infestantes de $H$. contortus. Les deux plantes sont plus efficaces sur $H$. contortus uniquement à une forte dose d'extrait des résidus lorsque le temps d'incubation est de 96 h. Ce travail conforte encore plus le choix des petits éleveurs du Bénin à traiter leurs animaux notamment les petits ruminants par l'usage des deux plantes. De plus ses différents résultats constituent des prémices dans le long processus de mise en place de Médicaments Traditionnels Améliorés (MTA).

\section{Remerciement}

Les auteurs sont reconnaissants au Projet Valorisation des Plantes locales pour l'Amélioration de la santé et de la Production des animaux d'élevage (VPMAP) en Afrique de l'Ouest financé par l'UEMOA à travers le Projet PAES Profondes gratitudes à l'endroit des reviewers pour leurs précieuses contributions au manuscrit.

\section{References:}

1. Adémola, I.O., Akanbi, A.I. \& Idowu, S.O. (2005). Comparative nematocidal activity of chromatographic fraction of Leucaena leucocephala seed against gastrointestinal sheep nematodes. Pharmacy and Biology, 43: 599- 604.

2. Aerts, R.J., Barry, T.N. \& McNabb, W.C. (1999). Polyphenols and agriculture: beneficial effects of proanthocyanidins in forages. Agriculture, Ecosystems \& Environment,75 (1-2): 1-12.

3. Alowanou, G.G., Olounladé, A. P., Koudandé, O. D., Babatoundé, S. \& Hounzangbé-Adoté, M. S. (2015). Effets de la digestion dans le rumen sur les propriétés anthelminthiques de Bridelia ferruginea, Mitragyna inermis et Combretum glutinosum. Science de la vie, de la terre et agronomie, 03(2): 50-56.

4. Andrea, B., Doeschl-Wilson, R., Davidson, J., Conington, T., Roughsedge, M.R. \& Hutchings, B.V. (2011). Implications of host genetic variation on the risk and prevalence of infectious diseases transmitted through the environment. Genetics, 188: 683-693.

5. Azando, E.V.B., Olounladé, A.P., Hounzangbé-Adoté, M.S. \& Hoste, H. (2011a). Effets anthelminthiques in vivo de la poudre de feuilles de Zanthoxylum zanthoxyloïdes et de Newbouldia laevis sur les nématodes parasites gastro-intestinaux des chevreaux Djallonké. 
International Journal of Biological and Chemical Science, 5 (3): 1054-1062.

6. Azando, E.V.B., Hounzangbé-Adoté, M.S., Olounladé, P.A., Brunet, S., Fabre, N., Valentin, A. \& Hoste, H. (2011b). Involvement of tannins and flavonoids in the in vitro effects of Newbouldia laevis and Zanthoxylum zanthoxyloides extracts on the exsheathment of third-stage infective larvae of gastrointestinal nematodes. Veterinary Parasitology; 180: 292-297.

7. Babatoundé, S. (2005). Etude et prédiction de la valeur alimentaire de graminées et de légumineuses fourragères en zone tropicale humide du Bénin. Thèse de doctorat Unique. Université de Liège-Gembloux Agro-Bio Tech (ULg) (Belgique).

8. Bruneton, J. (2009). Pharmacognosie-Phytochimie, plantes médicinales, 4e éd., revue et augmentée, Paris, Tec \& Doc - Éditions médicales internationales.1288pp.

9. D’Assonville, J.A., Janovsky, E. \& Versley, A. (1996). In vitro screening of Haemonchus contortus third stage larvae for ivermectin resistance. Veterinary Parasitol, 61:73-80.

10. Dédéhou, N.F. (2015). Utilisation de Parkia biglobosa et de Pterocarpus erinaceus dans la gestion du parasitisme à Haemonchus contortus chez le mouton Djallonké au Bénin. Thèse de doctorat en Sciences Agronomiques - Université d'Abomey-Calavi (Bénin).

11. Hassanpour, S., Eshratkhah, B., Sadaghian, M., Maherisis, N. \& Chaichisemsari, M. (2011). Relationship between plasma minerals and nematode infection load in Moghani ewes. Global Veterinaria, 6 (4):357-361.

12. Hoste, H., Torres-Acosta, J.F.J., Paolini, V., Aguilar-Caballero, A., Etter, E., Lefrileux, Y., Chartier, C. \& Broqua, C. (2005). Interactions between nutrition and gastrointestinal infections with parasitic nematodes in goats. Small Ruminant Research, 60, 141-151.

13. Hounzangbé-Adote, M.S., Paolini, V., Fouraste, I., Moutaïrou, K. \& Hoste, H. (2005a). In vitro effects of four tropical plants on three stages of the parasitic nematodes, Haemonchus contortus. Research in Veterinary Science, 78: 155-160.

14. Hounzangbé-Adoté, S., Paolini, V., Fouraste, I., Moutairou, K. \& Hoste, H. (2005b). In vitro effects of four tropical plants on the intestinal parasitic nematode, T. colubriformis. Journal of Helminthology, 79: 29-33.

15. Hounzangbé-Adoté, S., Zinsou, F.E., Hounkpe, V., Moutairou, K. \& Hoste, H. (2005c). In vivo effects of Fagara leaves on sheep infected with gastrointestinal nematodes. Tropical Animal Health and Production, 37: 205-214. 
16. Marie-Magdeleine, C., Boval, M., Philibert, L., Borde, A. \& Archimède, H. (2010). Effect of banana foliage (Musa x paradisiaca) on nutrition, parasite infection and growth of lambs. Livestock Science, 131(2-3): 234-239.

17. Minaflinou, S.S.I., Azando, E.V.B., Olounladé, A.P. \& HounzangbéAdotéM.S. (2015). Effets combinés des feuilles de Newbouldia laevis et de Zanthoxylum zanthoxylö̈des sur les nématodes parasites gastro-intestinaux des ovins Djallonké. International Journal of Biological and Chemical Science, 9(4): 2078-2090.

18. Molan, A.L., Duncan, A.J., Barry, T.N. \& McNabb, W.C. (2003). Effects of condensed tannins and crude sesquiterpene lactones extracted from chicory on the motility of larvae of deer lungworm and gastrointestinal nematodes. Parasitology International, 52 (3), 209-218.

19. Olounladé, A.P. (2005). Effets anthelminthiques des feuilles de Newbouldia laevis testées in vivo sur les nématodes gastrointestinaux (Haemonchus contortus et Trichostrongylus colubriformis) chez les moutons Djallonké. DEA, Université de Lomé (Togo).

20. Olounladé, P.A., Hounzangbé-Adoté, M.S., Azando, E.V.B., Tam Ha, T.B., Brunet, S., Moulis, C. \& Fabre, N. (2011). Etude in vitro de l'effet des tannins de Newbouldia laevis et de Zanthoxylum zanthoxyloïdes sur la migration des larves infestantes de Haemonchus contortus. International Journal of Biological and Chemical Science; 5: 1414-1422.

21. R Core Team. (2013). R: A language and environment for statistical computing. R Foundation for Statistical Computing, Vienna, Austria. URL http://www.R-project.org/.

22. Rabel, B., McGregor, P. \& Dough, G. (1994). Improved bioassay for estimation of effects of ovine gastrointestinal inhibitory mucus and on nematode larval migration anthelminthic. International Journal of Parasitology, 24: 671-676.

23. Satyavir, S. \& Gupta, S.K. (2010). A survey of anthelmintic resistance in gastrointestinal nematode in sheep of Haryana. Haryana Veterinary, 49: 25-28.

24. Wabo-Poné, J., Yondo, J., Fossi, T.O., Komtangi, M.C., BilongBilong, C.F. \& Mpoame, M. (2011). The in vitro effects of Chenopodium ambrosioides (Chenopodiaceae) extracts on the parasitic nematode Heligmosomoid esbakeri (Nematoda, Heligmosomatidae). Journal of Pharmacognosy and Phytotherapy, 3: 56-62. 